\title{
COMPARATIVE COMPUTATIONAL ANALYSIS OF A PUTATIVE TRANSCRIPTIONAL REGULATOR MAP_PRSO3010 AND ITS IMPLICATIONS IN THE PATHOGENESIS OF CROHN'S AND JOHNE'S DISEASES
}

\author{
ASIF HASSAN SYED1* \\ ${ }^{1}$ Department of Computer Science, Faculty of Computing and Information Technology, Rabigh, King \\ Abdulaziz University, Jeddah, Saudi Arabia \\ Email: shassan1@kau.edu.sa \\ Revised Octobar 2016 \\ ABSTRACT. The implications of specific biochemical and structural features of many \\ putative virulence factors in the pathogenesis of MAP in ruminant and human being \\ are relatively less explored and understood. In this background, the comparative \\ genomic and proteomic studies of MAP_PRSO3010, a putative protein shows that it \\ shares a sequential, physiochemical, structural and functional similarity with \\ Rv0757 (PhoP) protein, a virulent molecular determinant of Mycobacterium \\ tuberculosis (Mtb). Conserved Domain Database (CDD) and InterPro studies \\ demonstrate the presence of conserved domains namely Signal transduction \\ response regulator, receiver domain (REC) and Transcription regulatory protein, \\ C-terminal (Trans_reg_C) belonging to CheY-like and Helix Turn Helix (HTH) \\ superfamily, respectively in both MAP_PRSO3010 and Rv0757 proteins. These \\ domains are mainly involved in phosphorelay signal transduction and \\ transcriptional regulation in response to unfavorable environments within host \\ macrophages. Comparative in silico protein-protein interaction (PPI) studies also \\ showed the involvement of common interacting proteins namely $\mathrm{mtrA} / \mathrm{B}$ crucial for \\ the survival of Mtb within host macrophages. The predicted hypothetical model of \\ MAP_PRSO3010 protein provides an insight on the functional and structural \\ resemblance between the two proteins. The model quality and structure assessment \\ tools of Swiss-Model Server also validated the predicted hypothetical structure of \\ MAP_PRSO3010 protein. Thus, these results show a strong relevance of \\ MAP_PRSO3010 protein in MAP virulence.
}

Keywords: MAP_PRSO3010; Rv0757; Phosphorelay Signal Transduction; Transcriptional Regulator; Virulence factors; MAP; Crohn's and Johne's diseases.

1. Introduction. The role of MAP in the virulence of Johne's disease is well known [1] and very recently its relationship with Crohn's disease, an inflammatory gut infection, affecting humans had been discussed [2-3]; [4-5]. MAP is a successful intracellular pathogen and infects mainly macrophage cells of the gastrointestinal tract of humans and ruminants [1]. Though, only a little is known about the comprehensive mechanisms that regulate the expression of genes involved in the survival of species belonging to genus Mycobacterium within host macrophages, it is most likely that persistence of Mycobacterium sp. within host cells is contributed by signal transduction and transcriptional regulation [6]. The two-component regulatory systems in mycobacteria and lower eukaryotes have been instrumental in the survival of mycobacterial pathogens in the unfriendly environment in ruminants and human macrophages. These systems are involved in intricate signal transduction network that responds to environmental stimulus to cellular response via the response regulator, 
which are normally transcriptional regulatory proteins [8]. The transcriptional regulatory factor-PhoP of the "PhoP-PhoR two-component regulatory system" plays a key role in the survival and persistent intracellular growth and of $M t b$ in mouse and humans macrophages via the mechanism of gene regulation which is also essential for the biosynthesis of certain complex lipid critically important in Mtb pathogenesis [8-14]. In recent times, the majority of the disease mechanisms of MAP have been elucidated are based on the mode of diseases triggered by Mycobacterium avium ssp. avium (MAA) and Mtb [15]. Hence, a thorough comparative sequential, functional and structural study was conducted between "PhoP" a response regulatory constituent of the "two-component regulatory system" of $M t b$ and its ortholog present in the genome of MAP. The current comparative study was directed to provide an insight into the role of the putative gene MAP_PRSO3010 (phoP) in the pathogenesis and virulence of MAP in humans and ruminants, respectively. In this regard, the comparative genomic and proteomics analysis of Mtb phoP and MAP_PRSO3010 (phoP) show that the putative protein MAP_PRSO3010 ( $p h o P$ ) locus of MAP genome shares a sequential, structural and functional homology with $M t b$ phoP (Rv0757) protein. In silico protein-protein interaction studies shows that MAP_PRSO3010 (phoP) protein interacts with proteins namely mtrA/B which are important for intracellular growth and survival of $M t b$ in human macrophages. Since, MAP_PRSO3010 (phoP) a putative protein present a strong functional and structural correlation with $M t b$ PhoP protein, we propose that MAP_PRSO3010 a possible response regulatory component of the two-component regulatory system of MAP might play a significant role in the regulation and expression of key virulent genes involved in sustaining the growth of mycobacterium in the hostile environment of host macrophages and causing infections.

The paper is organized as follows. Section 2 is devoted for the screening of an ortholog of Rv0757 protein as well as comparative physio-chemical, structural and functional studies of the orthologs. The results and discussion of the screening process as well as comparartive fuctional and structural studies are represented in Section 3 and 4 respectively. Section 5 highlight the concluding remarks of the present research work.

2. Methods. In this section, we identify and comprehensively compare the physio-chemical, structural and functional similarity between the orthologs.

- Orthology prediction. EggNOG [16] an orthology prediction methodology employing Graph-based method was employed to search for an ortholog of the Rv0757 (phoP) gene in MAP genome. (http://eggnogdb.embl.de/\#/app/guided_search).

- Sequence retrieval. The GenInfo Identifier (GI) number and the sequences of the transcriptional response regulator PhoP from $M t b$ and its corresponding orthologous protein from MAP in FASTA format were attained from National Center for Biotechnological Information (NCBI) website. (http://www.ncbi.nlm.nih.gov/protein).

- ProtPram analysis. ProtPram tools such as Instability index [18], Grand Average of Hydrophobic index (GRAVY) [17] and aliphatic index [19] and from ExPASy portal (http://web.expasy.org/protparam/) were used to compare the physicochemical properties of the transcriptional response regulator phoP (Rv0757) of $M t b$ and its corresponding orthologous protein from MAP.

- Hydropathicity analysis. The Hydropathicity plot (http://web.expasy.org/protscale/) [20] was used to compare and predict the possible surface region of Rv0757 (phoP) and its orthologous protein from MAP.

- Protein interaction studies. STRING a database made from predicted and experimental proven protein-protein interactions were used to screen common interacting proteins partner of both MAP_PRSO3010 and Rv0757 proteins ([21]Szklarczyk et al. 2015). The present study will highlight the transcriptional regulatory role of MAP_PRSO3010 protein and its implication in the pathogenesis of MAP causative diseases. (http://smart.embl-heidelberg.de/smart). 


\section{- Sequence analysis}

- Domain analysis. InterPro [22] and CDD [23] protein sequence analysis and classification tools were used to identify domains and provide extensive annotations of Rv0757 (phoP) and its orthologous protein MAP_PRSO3010.

- Secondary, transmembrane and disorder studies. The secondary structural elements of MAP_PRSO3010 and Rv0757 proteins were compared using PDBsum database [24]. The Position Specific Iterated Protein Secondary Structure Prediction server (PSIPRED) was employed to compare the transmembrane topology [25] and disorder regions [26] of Rv0757 and its orthologous protein from MAP.

\subsection{Automated template based modeling and validation}

- Model building. The automated homology modeling program of the SWISS-MODEL server [27-30] was employed to generate a hypothetical 3D model of the MAP_PRSO3010 protein. The theoretical model of MAP_PRSO3010 was built using the three-dimensional coordinates of the crystal structure of Rv0757 (3r0j.1.B) protein using Modeller [31] and Promod-II [32].

- Hypothetical model quality evaluation. Evaluating the quality of the predicted model is of vital importance in homology-based modeling. Therefore, the reliability of predicted MAP_PRSO3010 protein 3D structure was evaluated using GMQE [27]. and QMEAN6 [33] score. The reliability index of GMQE ranges from 0 to 1 . The model is considered reliable if the score is closer to 1 . The QMEAN score of the MAP_PRSO3010 structure was assessed against highly refined and highly resolved reference crystallized structures of equal sizes and consequently, a QMEAN Z Score of each individual structural descriptors for the theoretical structure was estimated. The standard Z-Score for structures identical to that of reference structures is zero [34]. GROMOS [36] and ANOLEA [35] was employed for estimating the local quality of the model. The pseudo energy of the entire hypothetical model of MAP_PRSO3010 protein was estimated using DFIRE a global structure assessment tool of the Swiss-Model server. A lower pseudo energy value of any structure indicates that the structure is closer to its native conformation [37]. PROCHECK a software tool was employed to evaluate the stereochemical quality of the predicted hypothetical protein structure of MAP_PRSO3010. The PROCHECK program determines the geometrical similarity of the amino acids in the predicted hypothetical structure of MAP_PRSO3010 protein with well-defined highly resolved crystal structures [38].

3. Results. In this section, we describe the results of the software tools used for the screening as well as structural and functional characterization of the orthologs.

Orthology analysis. A putative protein MAP_PRSO3010 from MAP was identified as an ortholog of Mtb Rv0757 ( $p h o P$ ) protein from the eggNOG database. MAP_PRSO3010 protein shares a sequence similarity of $97.12 \%$ with a Rv0757 protein of $M t b$. Based upon its sequential similarity with Rv0757 (phoP) protein, MAP_PRSO3010 was predicted to be a response regulator (RR) factor of the "two-component regulatory system" of MAP.

ProtPram analysis of the orthologous proteins. The physiochemical analysis showed resemblance of several physical and chemical descriptors among Rv0757 and MAP_PRSO3010 proteins as tabulated in Table 1. Both MAP_PRSO3010 and Rv0757 are theoretically stable since their instability index is smaller than 40 . The instability index of protein above 40 is considered unstable in a test tube. The GRAVY value for both MAP_PRSO3010 and Rv0757 proteins are negative and this shows that on the whole, both the proteins are globular and hydrophilic by nature. The comparative aliphatic index shows that the relative volume of the aliphatic side chains (leucine, isoleucine, alanine, and valine) of both MAP_PRSO3010 and Rv0757 proteins are similar therefore both the orthologs, are equally stable to heat. 
Table 1. A comparative study of the computed physical and chemical parameters of ProtPram of the orthologous response regulators of MAP and Mtb

\begin{tabular}{llllll}
\hline Protein & $\begin{array}{l}\text { Grand Average } \\
\text { of } \\
\text { Hydropathicity } \\
(\text { GRAVY) }\end{array}$ & $\begin{array}{l}\text { Aliphatic } \\
\text { Index }\end{array}$ & $\begin{array}{l}\text { Instability } \\
\text { Index }(>>\text { Amino acid } \\
\text { 40 }\end{array}$ & $\begin{array}{l}\text { Protein } \\
\text { composition }\end{array}$ & $\begin{array}{l}\text { Molecular } \\
\text { weight } \\
\text { (Dalton) }\end{array}$ \\
& -0.181 & 99.09 & 35.12 & 247 & 27513.5 \\
Rv0757 (PhoP) & 98.60 & 38.60 & 239 & 26476.2 \\
\hline MAP_PRSO3010 & -0.152 & $98.60)$ & \\
\hline
\end{tabular}

Hydropathy analysis of the orthologous proteins. A detailed comparative hydropathy profile of both MAP_PRSO3010 and Rv0757 protein is shown in Figure 1a and 1b, respectively. The presence of a large number of negative peaks with a hydropathy score of -2.0 or more shows that most of the amino acids in these two orthologous proteins are located in the exterior region and are hydrophilic by nature. Both MAP_PRSO3010 and Rv0757 protein show a positive hydropathy score of +1.6 and above for at least six short stretches of amino acids indicating the presence of hydrophobic regions at these segments of the globular orthologous proteins.

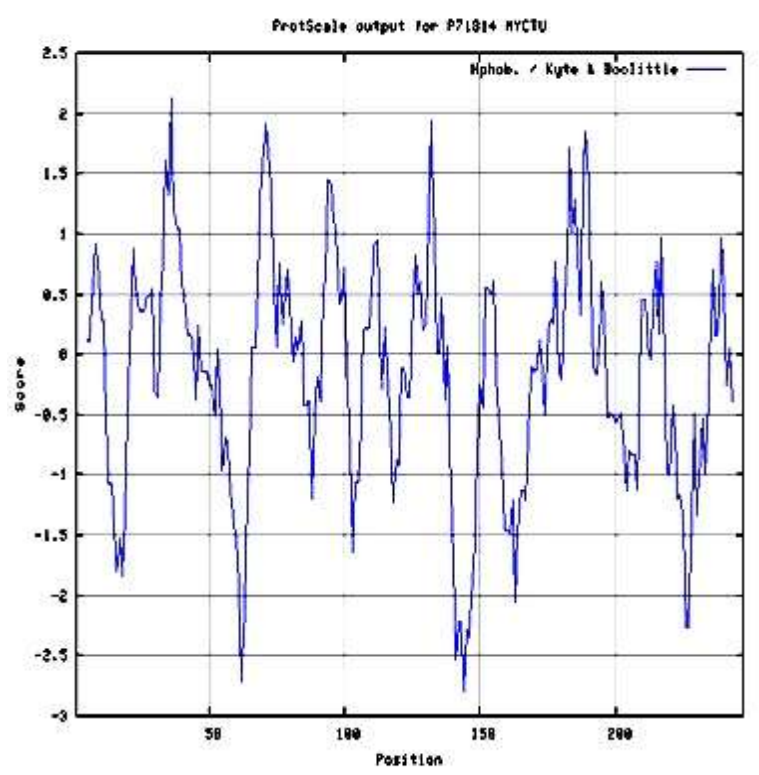

(a)

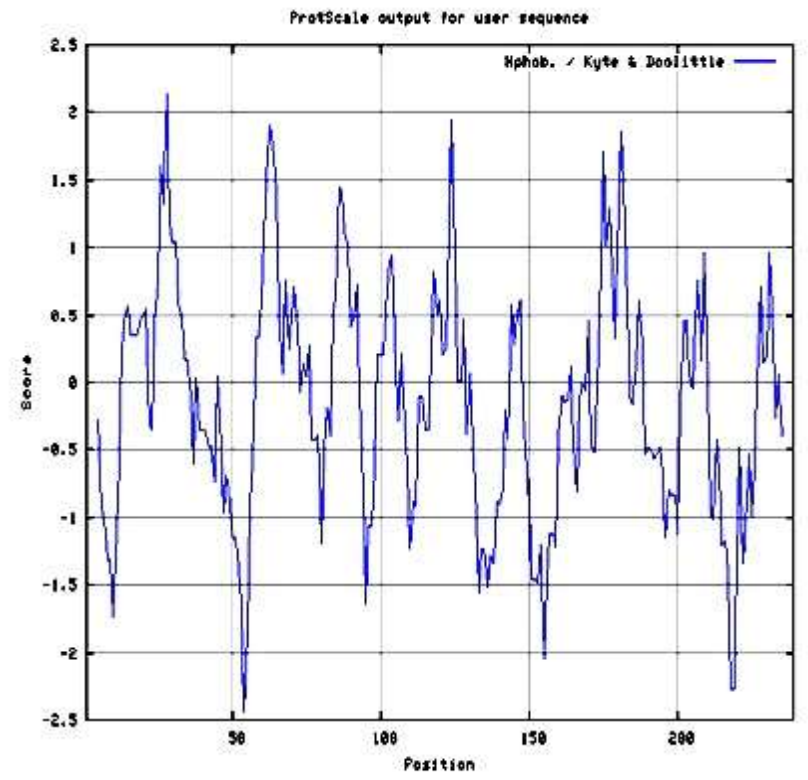

(b)

Figure-1: Pictorial representation of the hydropathy plot of (A) Rv0757 and (B) MAP_PRSO3010 proteins

Protein-protein interaction (PPI) studies of Rv0757 and MAP_PRSO3010 proteins. A functional protein associated network of both MAP_PRSO3010 and Rv0757 protein was compared to identify common interacting proteins crucial for the survival and multiplication of the bacterium within host macrophages. Figure $2 \mathrm{a}$ and $2 \mathrm{~b}$ shows the PPI profile of MAP_PRSO3010 and Rv0757 proteins, respectively. The comparative PPI analysis reveals that both MAP_PRSO3010 and Rv0757 proteins interact with the mtrB and SenX3 protein. 


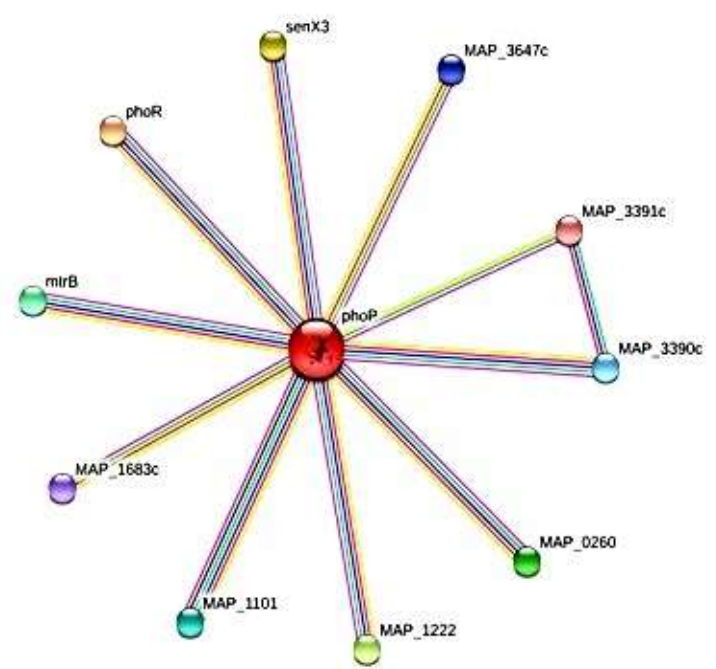

2A

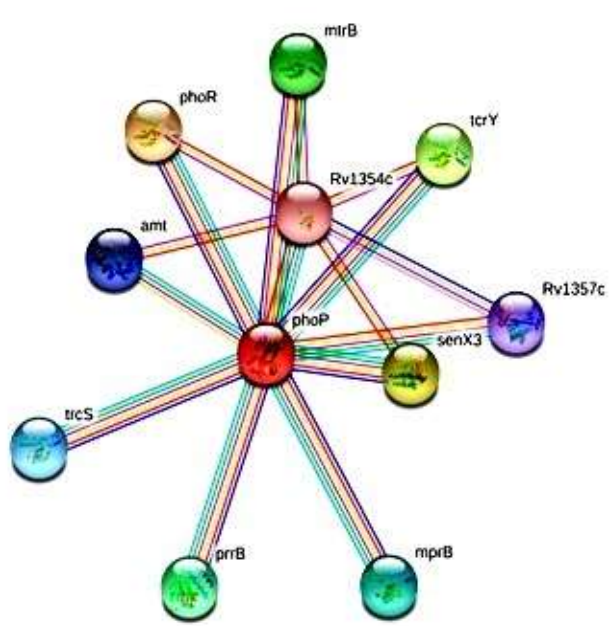

2B

Figure-2 (a-b): Pictorial representation of PPI of (A) MAP_PRSO3010 and (B) Rv0757 proteins using STRING

\section{Sequential, structural and functional investigation of orthologous proteins}

Domain analysis. The comparative domain architecture analysis showed that both MAP_PRSO3010 and Rv0757 proteins have similar domain organization and composition. The ProScan and CDD analysis of MAP_PRSO3010 protein sequence demonstrate the presence of domains namely the signal receiver domain, effector domain of response regulator and a DNA-binding response regulator. The summary of the domains of MAP_PRSO3010 and Rv0757 are tabulated in Table 2.

Table 2. Tabulation of domains present in both MAP_PRSO3010 and Rv0757 proteins

\begin{tabular}{llc}
\hline Name & Description & Interval \\
\hline CheY-like_superfamily & Signal receiver domain & $2-85$ \\
Sig_transdc_resp-reg_receiver & Signal transduction response regulator, receiver domain & $1-72$ \\
wHTH_DNA-bd_dom & Winged helix-turn-helix DNA-binding domain & $88-186$ \\
Sig_transdc_resp-reg_C-effctor & Signal transduction response regulator, C-terminal effector & $87-184$ \\
Sig_transdc_resp-reg_C & Signal transduction response regulator, C-terminal & $109-183$ \\
\hline
\end{tabular}

Secondary, transmembrane and disorder structure analysis. The comparative secondary and super-secondary structure analysis of MAP_PRSO3010 and Rv0757 proteins by PDBsum reveal that the organization and composition of the two orthologs are similar as shown in Figures 3a and 3b, respectively. 


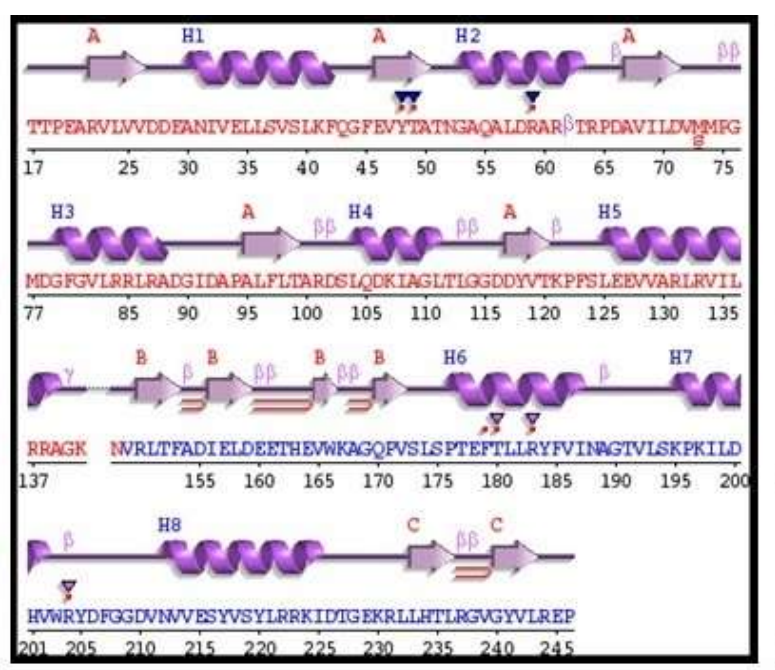

3A

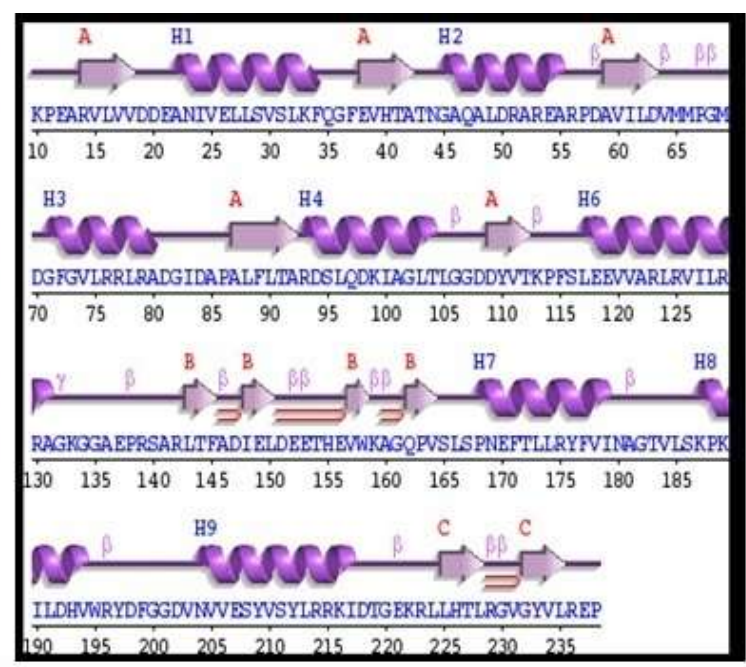

3B

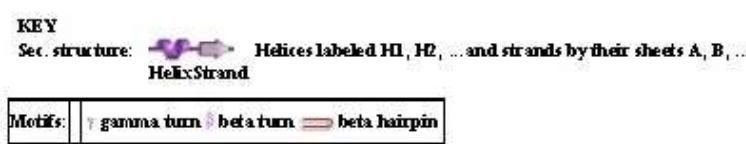

Figure-3: Comparative pictorial representation of secondary structure of (A) Rv0757 and (B) MAP_PRSO3010 proteins predicted by PSIPRED server

The percentage of the strand, alpha helix, 3-10 helices and other super secondary structures in the predicted model were $20.1 \%, 40.2 \%, 0.0 \%$ and $39.7 \%$, respectively. The prediction of transmembrane topology/helix and protein intrinsic disorder was performed using PSIPRED based protein sequence analysis workplace. The MEMSAT-SVM and MEMSAT3 software of PSIPRED workplace showed that both the orthologs MAP_PRSO3010 and Rv0757 proteins consist of the transmembrane helical region as shown in Figures $4 \mathrm{a}$ and $4 \mathrm{~b}$ for MAP_PRSO3010 protein and Figures 5a and 5b for Rv0757 protein, respectively.

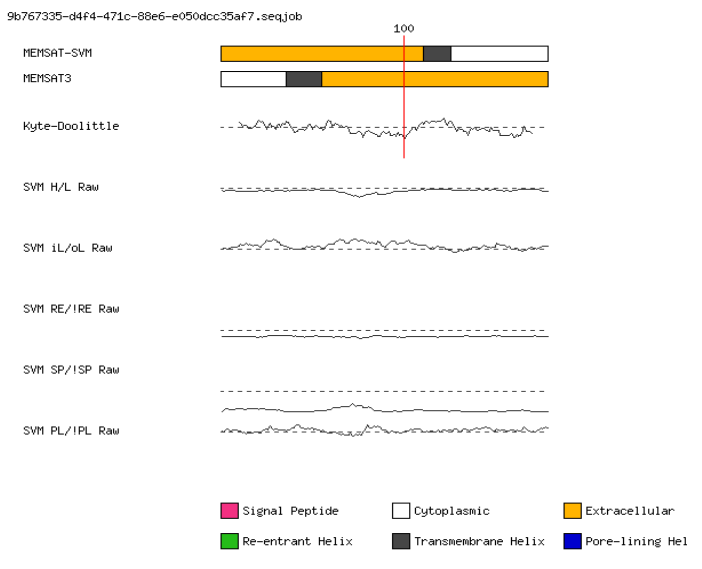

Figure-4a

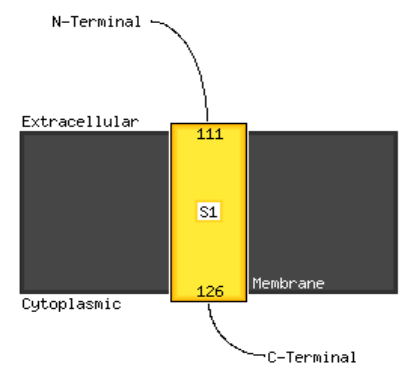

Figure-4b

Figure-4(a-b): Schematic diagrams of the (a) MEMSAT3 and (b) MEMSATSVM predictions for MAP_PRSO3010 protein sequence 


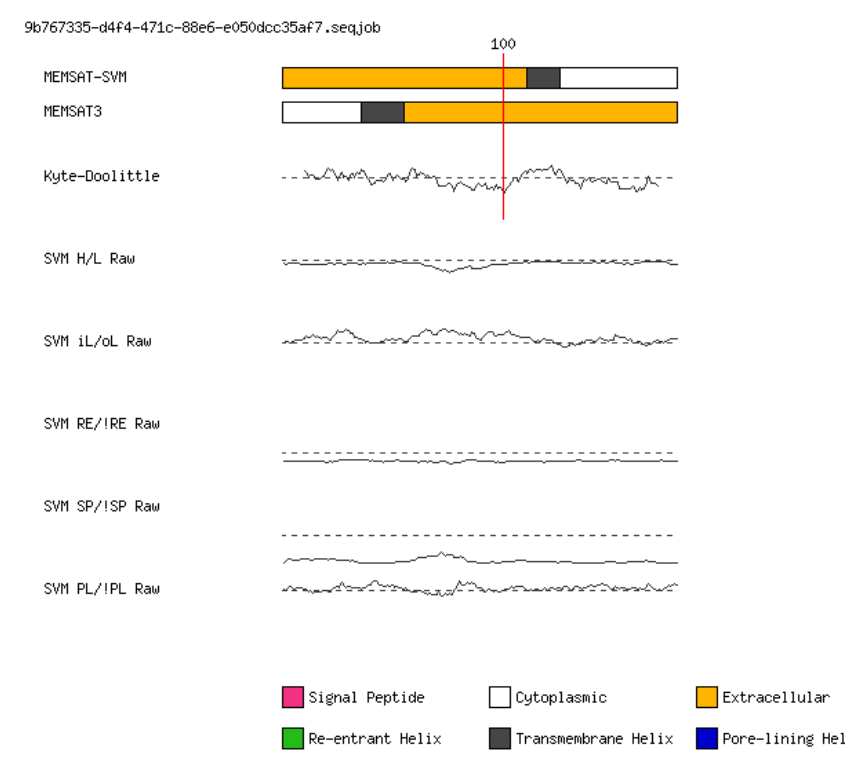

Figure-5a

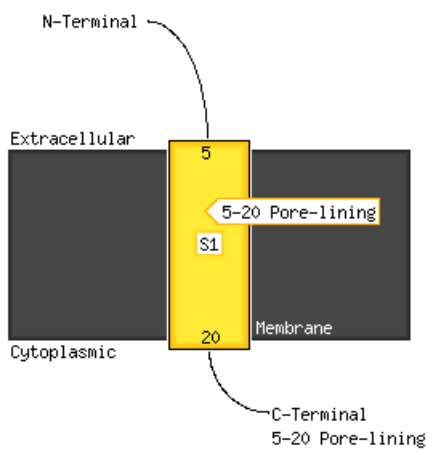

Figure- 5b

Figure-5 (a-b): Schematic diagrams of the (a) MEMSAT3 and (b) MEMSATSVM predictions for Rv0757 protein sequence

The topology analysis of Rv0757 protein shows that the N-terminal of the protein is located in the extracellular region; the $\mathrm{C}$-terminal region is positioned in the cytoplasmic region and a transmembrane pore-lining helical region ranging from 5-20 amino acids at the N-terminal region of the protein. Contrary, MAP_PRSO3010 protein showed that the presence of a transmembrane pore-lining helical region in the C-terminal region of 111-126 amino acids. The N-terminal region of the MAP_PRSO3010 protein was located in the extracellular region while $\mathrm{C}$-terminal at the cytoplasmic end. N-terminal signal peptide sequences were not detected in both MAP_PRSO3010 and Rv0757 proteins. Disordered region for MAP_PRSO3010 protein is located at the N-terminal region from 2 to 8 and 144 to 149 amino acid position of the protein sequence. While for Rv0757 the disordered region is located at both the N-terminal (11-32 a.a and 90-99 a.a) and the C-terminal region (194-199 a.a) of the protein sequence.

\section{Result of homology modeling using Swiss-Model Server}

Template search and homology modeling. HHBlits and BLAST alignment tools were used to search for evolutionarily related structures matching the query sequence using the library of templates from SWISS-MODEL server (SMTL version 05-05-14, PDB release 25-04-2014). A predicted protein structure of the MAP_PRSO3010 protein was made using the three-dimensional coordinates of two-component system response transcriptional positive regulatory protein PhoP (PDB ID: 3r0j.1.B) as a template having a sequence identity of 90.12 percentage with the query protein sequence (MAP_PRSO3010). The summary of the template (3r0j.1.B) protein structure and the predicted 3D model of MAP_PRSO3010 proteins are tabulated in Table 3. The predicted 3D structure of an MAP_PRSO3010 protein is shown in Figure 6 and the superimposed structures of the crystal structure of Rv0757 and theoretical 3D structure of MAP_PRSO3010 proteins is shown in Figure 7. Structure superimposition studies show a significant homology of the secondary structure conformation between the X-ray crystal structure of Rv0757 and the predicted protein structure of MAP_PRSO3010 proteins, respectively. 
Table 3. Summary of the 3D model of MAP_PRSO3010 protein generated by Swiss-Model Server

\begin{tabular}{|c|c|c|c|c|}
\hline Template & $\begin{array}{l}\text { Predicted } \\
\text { Model } \\
\text { Structure }\end{array}$ & Built with & $\begin{array}{l}\text { Sequence Identity between Rv0757 } \\
\text { (Template) and } \\
\text { MAP_PRSO3010(Query) proteins }\end{array}$ & Description \\
\hline 3r0j.1.B & $\sqrt{\frac{6}{6}}$ & $\begin{array}{l}\text { ProMod } \\
\text { Version } \\
3.70\end{array}$ & $90.12 \%$ & $\begin{array}{lr}\text { Possible Two } & \text { Component } \\
\text { System } & \text { Response } \\
\text { Transcriptional } & \text { Positive } \\
\text { Regulator PhoP } & \end{array}$ \\
\hline
\end{tabular}

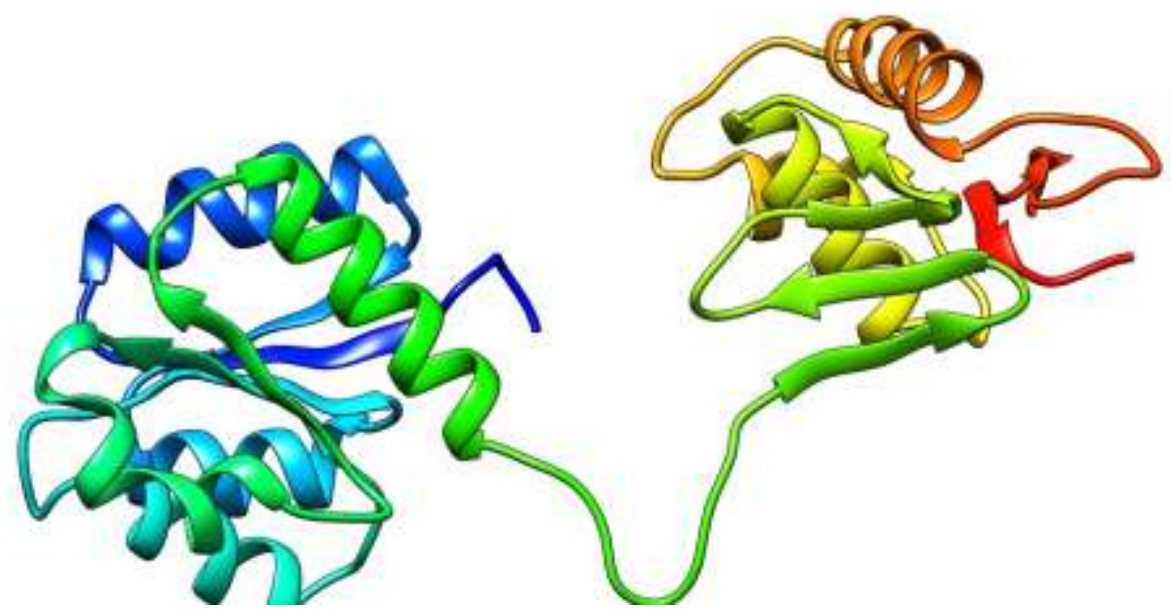

Figure-6: Shows the 3D protein structure of MAP_PRSO3010 generated by automated homology modeling of Swiss Model Server

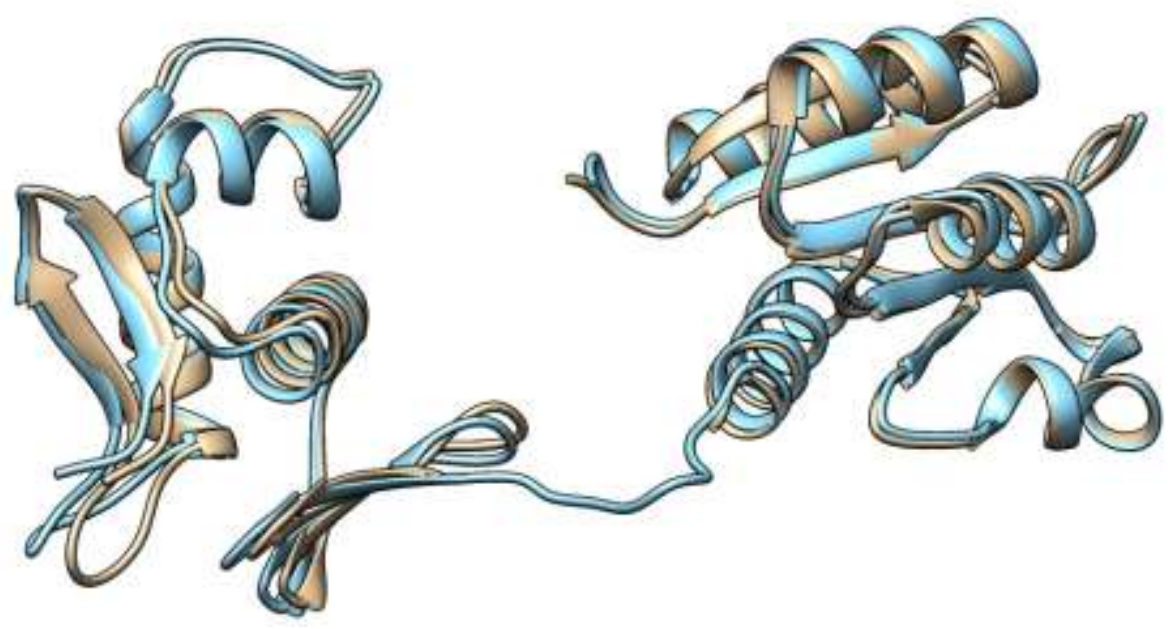

Figure-7: Superimposition of the predicted structure of MAP_PRSO3010 protein (blue) and the X-ray crystal structure of the Rv0757 protein. 


\section{Model quality Assessment of MAP_PRSO3010 protein three-dimensional structure}

Local model quality estimation. The local per-residue quality estimation of the predicted model based on QMEAN scoring function is depicted by the residue error plot of an MAP_PRSO3010 protein sequence as shown in Figure 8a. Small structural inaccuracy values for a majority of position in the predicted model of MAP_PRSO3010 protein sequence were observed indicating the reliability of predicted 3D model of the MAP_PRSO3010 protein. Estimated per residue inaccuracy of the predicted model was also visualized using a color gradient ranging from blue (most reliable region) to red (highly unreliable region). Since the majority of MAP_PRSO3010 protein sequence lie in the blue region, therefore, the predicted model of the MAP_PRSO3010 protein is expected to be stable and reliable. Figure 8b graphically represents the packing quality of the predicted model using ANOLEA and it was observed that majority of the amino acids on the B-chain of MAP_PRSO3010 protein have negative values represented in green color and only a small portion of the sequence is represented in red color (positive energy). Therefore, it can be presumed that most of the amino acids in MAP_PRSO3010 protein have favorable energy environment along the protein sequence. The confirmation of the individual residues of MAP_PRSO3010 protein sequence was evaluated using GROMOS and it was recognized that most of the amino acid possess high negative energy values indicating favorable energy environment as shown in Figure 9.

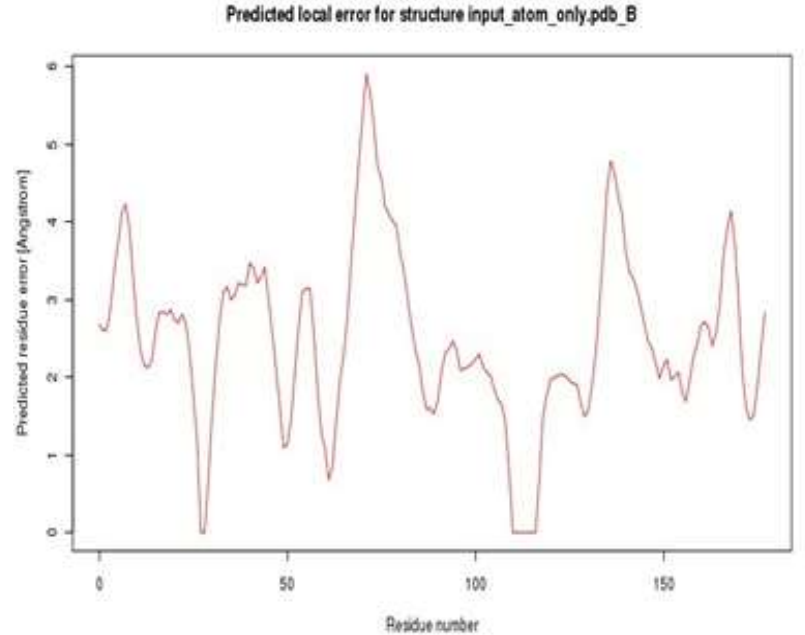

Figure-8(a)

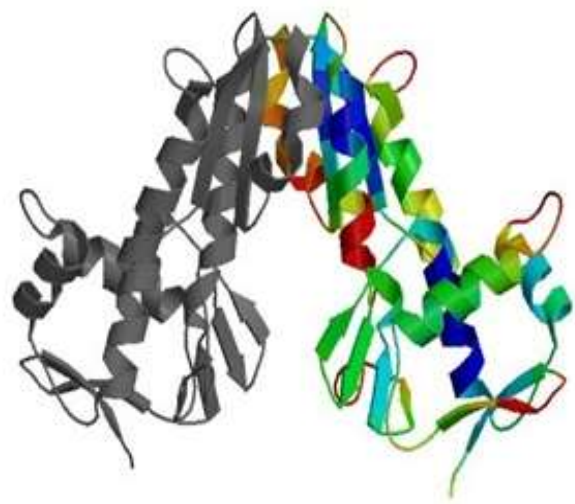

Figure-8(b)

Figure-8: (a) Residue error plot of the predicted structure of MAP_PRSO3010 protein (b) Estimated per residue inaccuracy of the predicted model of MAP_PRSO3010 protein visualized using color gradient from blue (highly reliable region) to red color (highly unreliable region).

Global model quality estimation. A QMEAN 6 score was employed to evaluate the global quality of the predicted model. Each of the amino acid in the MAP_PRSO3010 protein sequence is assigned a reliability score ranging from 0 to 1 . A score closer to 1 indicates higher reliability and similarity of residues to the native structures. A QMEAN 6 score of 0.641 was observed showing that predicted model is reliable. QMEAN Z-score measures the relative "degree of nativeness" of the predicted structure of MAP_PRSO3010, when compared to the experimental X-ray structures of similar size, was calculated and was found be -1.309. The Z-score of each structural descriptors, as well as the global QMEAN Z-score of the predicted protein structure of MAP_PRSO3010, are illustrated in Figure 10a and 10b, respectively. The accuracy of the hypothetical protein model built using the three dimensional coordinates of Rv0757 protein as template was evaluated using GMQE which considers the properties obtained from the target-template alignment as well as a QMEAN6 score of the predicted protein structure to improvise the credibility of the model. The resulting GMQE value is represented as a score ranging from 0 (unreliable) to 1 (reliable). A GMQE score of 0.97 was obtained for the predicted structure of MAP_PRSO3010 protein reflecting upon the accuracy of the model built using Swiss-Model Server. The nonbonded atomic interactions in the predicted model were also evaluated using DFIRE. A lower DFIRE energy value -458.78 for the predicted model of MAP_PRSO3010 protein was observed which indicates that the predicted model is closer to its native confirmation. 


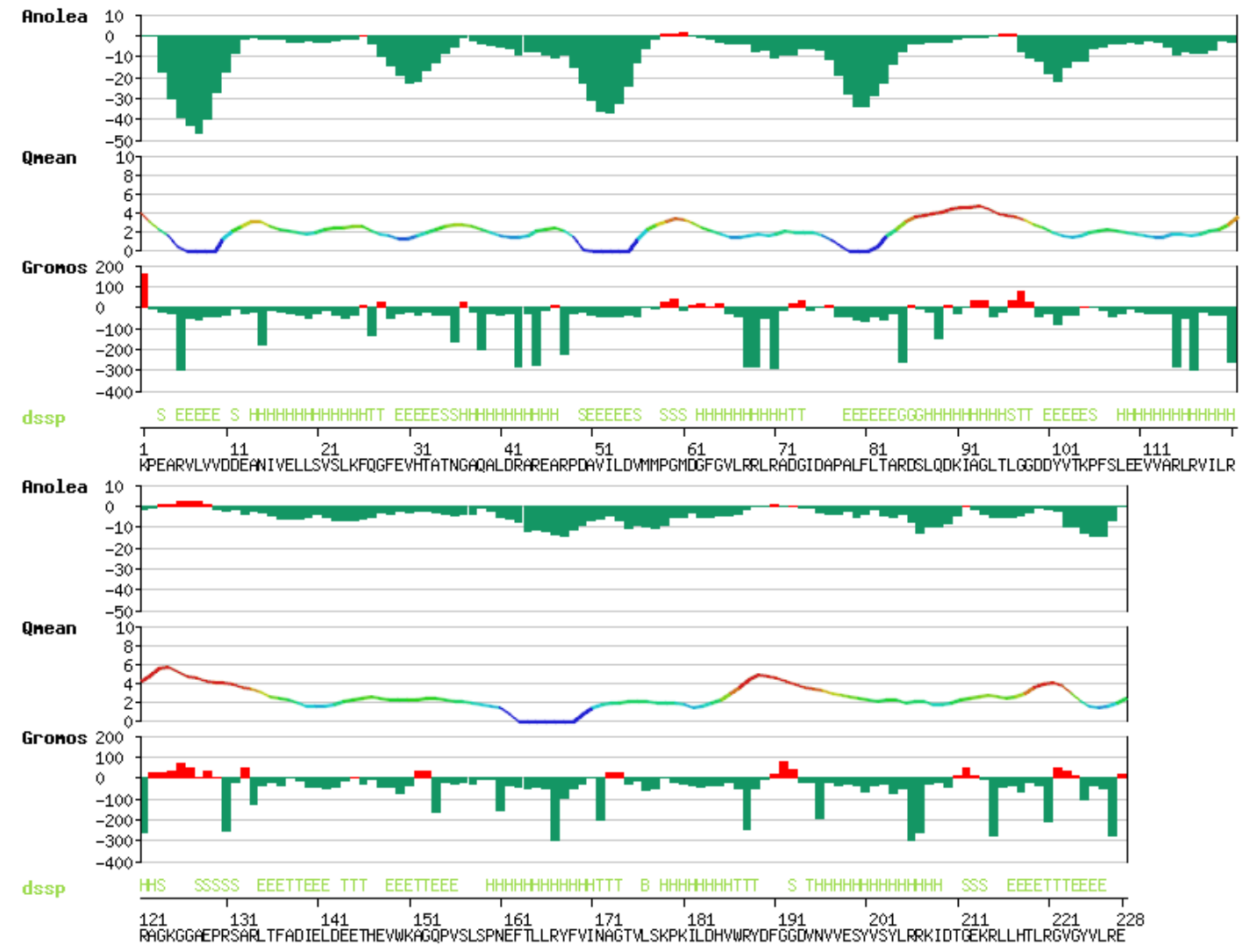

Figure-9: Graphical representation of ANOLEA, QMEAN and GROMOS analysis of MAP_PRSO3010 protein model

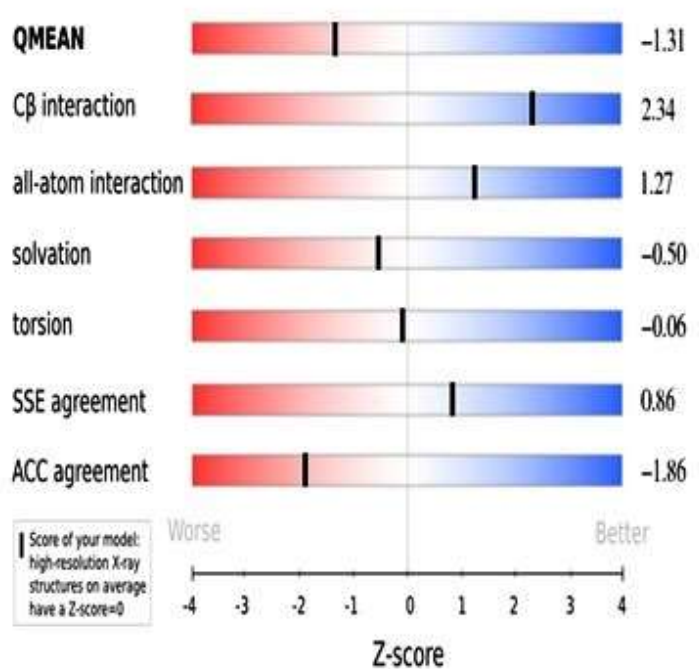

Figure-11(a)

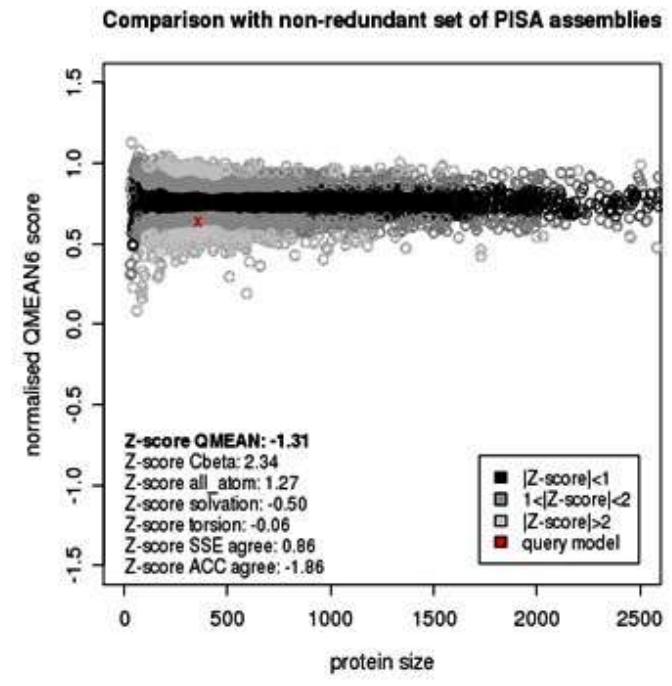

Figure-11(b)

Figure-11: Shows the Z-Score analysis of (a) Structural descriptors of QMEAN6 score and (b) 3D model of MAP_PRSO3010 protein generated by automated homology modeling 


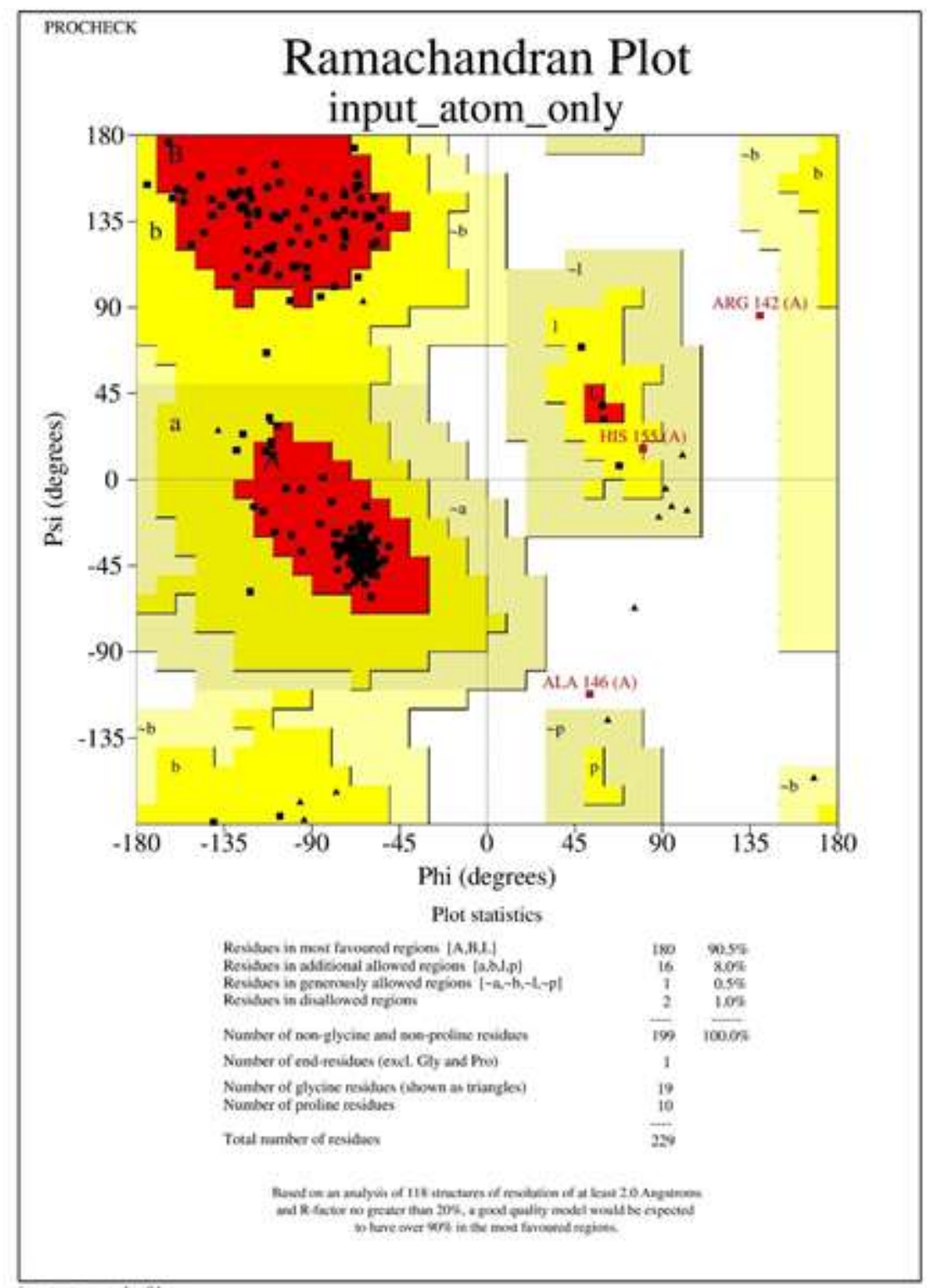

Figure-12: Stereochemical estimation of the predicted model of MAP_PRSO3010 protein structure using the Ramachandran plot of PROCHECK Server

\section{Structure Assessment of the predicted model of MAP_PRSO3010 protein}

Protein stereochemistry and structural motif \& promotif analysis. The "stereochemical quality" of the predicted model of MAP_PRSO3010 protein was evaluated. Structural validation of the predicted model using Ramachandran plot of PROCHECK suite showed satisfactory results. For the predicted 3D structure of MAP_PRSO3010 protein, $90.5 \%$ of the residues lie in the core region, $8 \%$ in allowed region, $0.5 \%$ in the generally allowed region and only $1 \%$ ( 3 labeled residues out of 227) of the amino acids were present in the disallowed region of the Ramachandran plot as shown in Figure 12.

The PROCHECK geometrical parameters specifically Chi1-chi2, Main chain of the predicted model of MAP_PRSO3010 protein were estimated and found worthy of future investigation. The chi1-chi2 plot showed only one residue out of 135 show unfavorable conformations (score < -3.00). Similarly, all the stereochemical parameters of Main chain and side chain were found to less deviate from the favorable confirmation of the reference structures at two angstroms or better. Likewise, other geometrical parameters namely main chain bond length (BL), bond angle (BA) and planar groups of the residues were within limits as 
compared to the reference structures and only $1.1 \%$ of the bond angle and $8.2 \%$ of the planar groups were found be in unfavorable confirmation (above limit). A summary of the stereochemical quality estimation of the MAP_PRSO3010 protein model is represented in Figure 13.

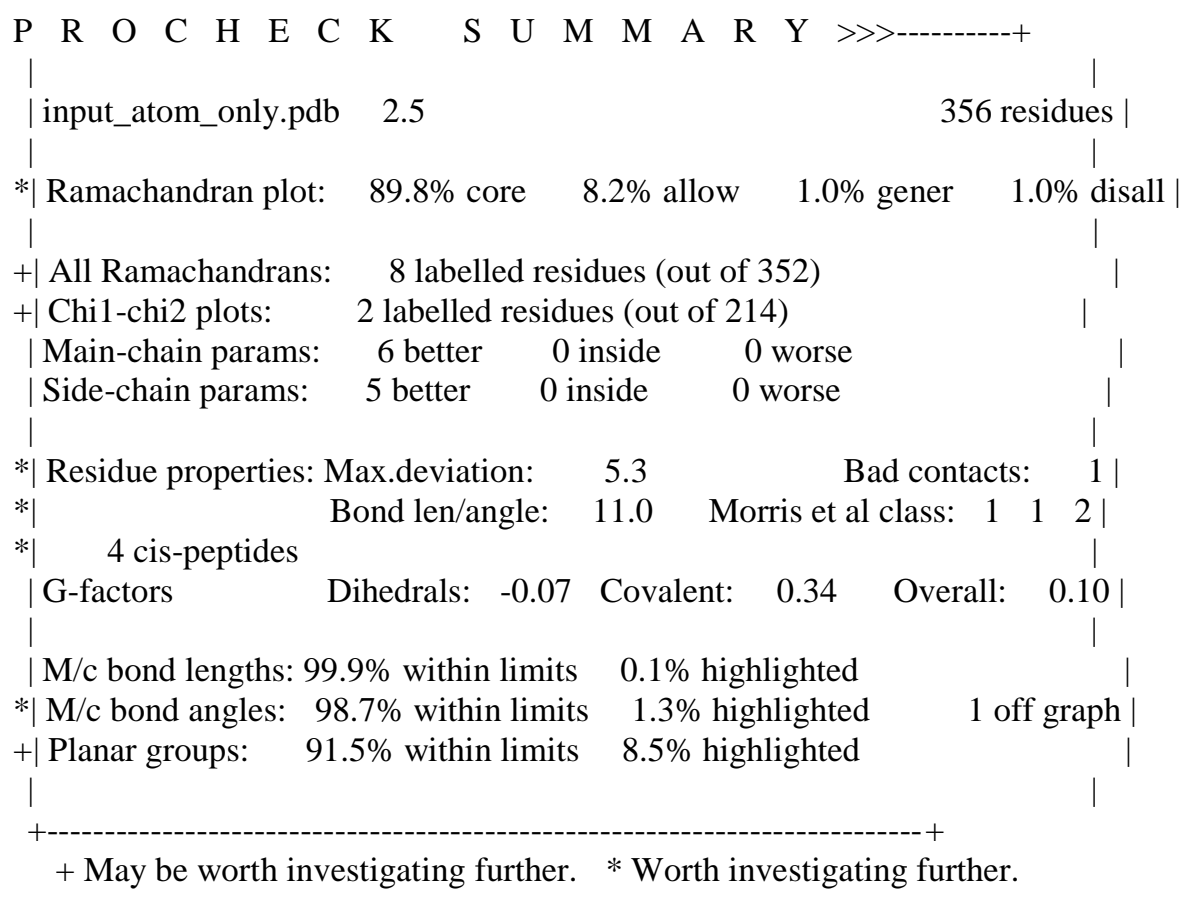

Figure-13: Summary of PROCHECK analysis of theoretical structure of MAP_PRSO3010 protein generated by the automated homology modeling mode of SWISS-MODEL Server

The motif analysis of the predicted model of MAP_PRSO3010 protein using PDBsum demonstrates the presence of dimerization interface (polypeptide interface) at the N-terminal segment of signal receiver domain (REC). The dimerization interface of MAP_PRSO3010 protein structure involves $\alpha 4-\beta 5-\alpha 5$ ranging from 86-93 a.a ( $\alpha 4), 95-105$ a.a ( $\beta 5)$ and 105-113 a.a ( $\alpha 5)$, respectively. Additionally, the presence of a typical fold, the winged helix-turn-helix (wHTH) in the DNA-binding region of the trans_reg_C domain was detected. The wHTH fold in the trans_reg_C domain of MAP_PRSO3010 protein structure starts with four antiparallel $\beta$ strands, where strand $1 \& 2$ and $3 \& 4$ are connected by a short $\beta$-strand while strand $3 \& 4$ are connected by a $\beta$-hairpin super secondary structure. The four antiparallel strands are further connected with a three-helical bundle of $\alpha$-helices. The three helices are connected to each other through short $\beta$-turn and further flanked at the $\mathrm{C}$-terminal by two short antiparallel $\beta$-strand connected together by a small $\beta$-hairpin structure (winged motif) as shown in Figure 3b. The first helix of wHTH fold is important for packing of other domains in three-dimensional space and the second and third helix along with the flexible long loop between them forms the HTH motif, with the third helix, forms the recognition helix important for recognition and interaction with the major groove of DNA.

4. Discussion. In this section, we dicuss the results of the software tools used for the screening as well as structural and functional characterization of the orthologs. We performed a comprehensive comparative genomic and proteomic study of a two-component system response transcriptional positive regulator PhoP of H37Rv in MAP genome and proteome. This study was undertaken to identify an ortholog gene of Rv0757 $(p h o P)$ in MAP genome since the PhoP protein of $M t b$ has a major involvement in the pathogenesis and virulence of $M t b$ in humans. This study will provide an insight into the functional role of the orthologous gene of Rv0757 (phoP) in the pathogenesis and virulence of MAP in human and ruminants. In this regard, our analysis led to the identification of a putative gene named MAP_PRSO3010, which share a $90.12 \%$ homology with a two-component response transcriptional regulatory protein $\mathrm{PhoP}$ of $\mathrm{H} 37 \mathrm{Rv}$ genome. PhoP in association with PhoQ a "two-component regulatory system" regulon is encoded during the initial stages of 
infestation of human macrophages and is essential for early intracellular growth and further multiplication in host macrophages, suggesting its importance in the establishment of infection and viability of the bacterium within host macrophages [14]. From the results of eggNOG and gapped BLAST, it was confirmed that MAP_PRSO3010 protein has a sequential homology with the crystallize structure of Rv0757 (PhoP) protein obtained from $\mathrm{H} 37 \mathrm{Rv}$ a pathogenic strain of $M t b$. Therefore, we performed a comparative proteomic; structural and functional study of MAP_PRSO3010 and Rv0757 (phoP) proteins to get an insight into the role of MAP_PRSO3010 protein in the persistence and virulence of MAP in ruminants and humans.

The comparative analysis of GRAVY by ProtParam (Table 1) shows that both MAP_PRSO3010 and Rv0757 $(p h o P)$ proteins are hydrophilic thereby rendering it soluble. The aliphatic index shows that both MAP_PRSO3010 and Rv0757 proteins have a higher aliphatic index. This signifies that both Rv0757 and MAP_PRSO3010 proteins are thermostable and can maintain their active conformation even at higher temperatures. The result of the instability index reveals that both MAP_PRSO3010 and Rvo757 (phoP) proteins are stable under in vitro conditions. These results show that sequence similarity is seen between MAP_PRSO3010 and Rv0757 ( $p h o P$ ) genes correlate with their physicochemical properties in the encoded proteins. The hydrophilic and hydrophobic contours of both MAP_PRSO3010 and Rv0757 proteins were comparable to the protein sequences. The hydropathy profile generated using the Hydropathicity plot by ExPASy ProtScale for MAP_PRSO3010 and Rv0757 ( $p h o P$ ) proteins may be used to identify the highly antigenic region with high hydrophilicity index for designing antigenic peptides and assess the antigenic potency of these peptides in stimulating a humoral and cell-mediated immune response. The PPI network studies of both MAP_PRSO3010 and Rv0757 ( $p h o P$ ) proteins showed that both the orthologs interact with $m t r B$ and sen $X 3$ proteins. Individually $m t r B$ and $\operatorname{sen} X 3$ are two-component sensor histidine kinase and members of two-component regulatory system $m t r A-m t r B$ and $\operatorname{sen} X 3-r e g X 3$, respectively. $m t r B$ and $\operatorname{sen} X 3$ proteins function as a protein kinase that phosphorylates $m t r A$ and $r e g X 3$, respectively in response to environmental stimulus. MtrA is an essential response regulator in Mtb and have a vital role in the viability of the bacterium within host macrophages [39-41]. Similarly, senX3-regX3 is required for Mtb virulence and evasion of the immune response within human macrophages [42-43]. The intracellular growth expression profiles of Mtb two-component regulatory genes show that MtrA is constitutively expressed while Rv0757 and senX3-regX3 show differential expression during different stages of post infection [44]. Since both $m t r A$ and senX3 share a sequential similarity with $m t r A$ and senX3 of MAP, therefore, we can predict that this PPI in MAP will also be important for the survival and consequently the propagation of the bacterium in host macrophages.

The ProScan and CDD domain study for both MAP_PRSO3010 and Rv0757 (phoP) proteins show that they possess common domains namely a signal receiver domain at the $\mathrm{N}$-terminal and an effector domain with DNA-templated transcriptional regulator activity at the C-terminal region of PhoP protein (response regulator). The receiver domain of the PhoP protein a member of the CheY superfamily receives the signal from a histidine protein kinase the sensor partner, in the bacterial two-component signaling system. The signal receiver domain contains a conserved active site consisting of eight conserved residues aspartic acid (Asp), glutamic acid (Glu), aspartic acid (Asp), asparagine (Asn), serine (Ser), valine (Val), lysine (Lys), proline (Pro) where Pro direct the side chain of Lys toward the active site and which eventually form a salt-bridge with the phosphorylation site consisting of one conserved residue aspartic acid (Asp). Asn, Ser, and Val are implicated in the activation process. The phosphoacceptor site (Asp) is phosphorylated by a histidine kinase homolog and upon phosphorylation forms a homodimer through the intermolecular recognition site consisting of five conserved residues methionine (Met), proline (Pro), valine (Val), glutamic acid (Glu) and asparagine (Asn) and dimerization interface (polypeptide interface) consisting of three conserved residue lysine (Lys), proline (Pro) and valine (Val) at the N-terminal region of REC. Since Rv0757 and MAP_PRSO3010 protein share sequential homology at the dimerization interface, it can be reasoned out that the dimerization interface in MAP_PRSO3010 protein predicted structure could also be classified into architecture-3-Layer (aba) Sandwich, class-alpha beta and topology-Rossman fold. Another important domain important for responding to the stimulus received is the DNA-templated transcription regulation domain (trans_reg_C) of RR. The effector domains C-terminal region comprises of a wHTH motif involved in the DNA-binding activity. The binding motif of effector domain binds to the alpha and sigma subunit of DNA and RNA polymerase through the conserved binding site namely threonine (Thr), serine (Ser), arginine (Arg), serine (Ser), valine (Val), methionine (Met), valine (Val), threonine (Thr) and glycine (Gly). These domains in Rv0757 protein are involved in phosphorelay signal transduction as well as modulation of the frequency or rate of cellular DNA-templated transcription of more than 110 genes leading to virulence and pathogenesis of tuberculosis in humans (Engohang-Ndong and Smith 2007). Since MAP_PRSO3010 protein shares a sequential and 
secondary structure topology similarity with Rv0757 protein, therefore, we can postulate that MAP_PRSO3010 protein will also be involved in the DNA-templated regulation of various genes in MAP leading to its virulence in ruminants and humans.

We also compared the secondary structure, transmembrane helix and disordered region of MAP_PRSO3010 and Rv0757 proteins using PDBsum and PSIPRED server. The comparative profiling of secondary structures of MAP_PRSO3010 and Rv0757 protein shows a greater amount of similarity in the location and amount of secondary structure (alpha helix and beta sheet) in both the proteins. This signifies the structural homology of the structural elements both the proteins. Likewise, the similarity in term of the presence of disorder and the transmembrane region was also observed. The presence intrinsically disordered regions signify the signaling and regulatory functional role of Rv0757 and MAP_PRSO3010 proteins since the disorder region enable interactions of these proteins with proteins from multiple pathways. This leads to the upregulation and downregulation of various genes involved the virulence of $M t b$ in humans [45-47]. Both MAP_PRSO3010 and Rv0757 protein show the presence of a series of amino acids having the propensity to form alpha-helix, and also lining the pore of the transmembrane at the N-terminal and C-terminal of MAP and $\mathrm{H} 37 \mathrm{Rv}$ bacterium. The presence of $\mathrm{N}$-terminal and the $\mathrm{C}$-terminal transmembrane region is vital in anchoring the proteins to the outer membrane of the bacterium. This anchoring activity of the transmembrane helices of MAP_PRSO3010 and Rv0757 protein might be useful for signal transduction and transcriptional regulation of other genes involved in the survival and propagation of the bacterium within host macrophages. For the given target protein MAP_PRSO3010, a library of experimental protein structures was explored to detect templates with highest identity or homology. Crystal structure of the Rv0757 protein (3ROJ) was selected to build a theoretical three-dimensional model of the MAP_PRSO3010 protein. The template structure (3ROJ) was screened based on its highest sequential homology with MAP_PRSO3010 (query) protein sequence. The superimpositions studies also showed significant secondary structural homology between the template (3ROJ) and the predicted structure of the MAP_PRSO3010 protein. Since the evaluation of the predicted structure is critically important in homology modeling therefore upon generation of the predicted protein structure of the MAP_PRSO3010 protein, the resulting model structure was validated using both local (Anolea, GROMOS, and QMEAN) as well as global (QMEAN6 Score and DFIRE) assessment tools of the swiss-model server. The local QMEAN score was calculated for each residue of the model and plotted using residue error pot. Mostly smaller values of structural inaccuracy were observed for each of the residues of the protein model except at certain regions of the protein sequence the QMEAN local value were larger this clearly signify that overall the predicted model of the MAP_PRSO3010 protein is reliable. The estimated per residue inaccuracy was visualized using a gradient of color ranging from blue to red, where in the case of MAP_PRSO3010 protein mostly all the residues lie in the blue region (more reliable region) while only a few were in the red region (potentially unreliable region) of the color gradient. The application of graphical plot of atomic mean force potential (ANOLEA) and empirical force field energy (GROMOS) to evaluate the local quality of the predicted protein structure of MAP_PRSO3010 showed that the conformational energy for each amino acid along the protein sequence lies mostly in the favorable energy (higher negative value) represented by green color in the plot. However, only a few amino acids of the protein structure were localized in the unfavorable energy environment (positive value) as represented by red color in the graphical plot of Anolea and GROMOS. These results of structural validation are encouraging since the estimated local quality of the theoretical structure of MAP_PRSO3010 protein were satisfactory.

Once the model is built using swiss-model server GMQE a global quality estimation tool which reflects the expected accuracy of the predicted protein structure of a value between zero and one. The GMQE score of MAP_PRSO3010 protein model was predicted to be 0.97 . Therefore higher fractional value (closer to 1)indicates higher accuracy and reliability of the MAP_PRSO3010 protein model built using the crystal structure of the RV0757 protein as a reference template. QMEAN6 a composite scoring function to evaluate the global quality and reliability of the predicted protein structure of MAP_PRSO3010 with values ranging from 0 (most unreliable) to 1 (highly reliable). Since the QMEAN6 Score of the predicted structure of the MAP_PRSO3010 protein was 0.641, therefore, the protein structure built using the template structure of Rv0757 protein is reliable and possibly the predicted structure of MAP_PRSO3010 protein is close to its possible native confirmation. The QMEAN Z-score of the predicted hypothetical structure is the composite score of the pseudo energies of six structural descriptors. The Z-score provides an estimation of the amount of deviation of the individual structural descriptor of a particular predicted model when compared to the reference X-ray crystal structures of similar sizes. The Z-score also compare the quality of the predicted structure with that of the experimental structure of similar size with predicted structures of low quality are 
likely to have greater negative QMEAN Z-scores 34]. The study of Z-scores for each geometrical descriptors will contribute in identifying the geometrical parameter accountable for the observed negative QMEAN Z-score. Since, the QMEAN Z-score of all except C $\beta$ interaction, all-atom interaction, and solvent accessibility agreement were approximately close to an ideal value (zero) the global QMEAN score of the predicted structure of MAP_PRSO3010 protein shows a negative standard deviation of one. A deviation of negative one is theoretically considered fair and the predicted structure can be used for further computational analysis ([34]. The pseudo energy of the entire three-dimensional model of MAP_PRSO3010 protein was assessed. The lower energy (-458.78) value obtained indicates that the predicted protein structure of MAP_PRSO3010 is of good quality and closer to native confirmation [37].

The geometry of the individual amino acid residues in the predicted protein structure of MAP_PRSO3010 was compared with the stereochemical parameters obtained from well-defined, high-resolution experimental structures of similar size using the PROCHECK suite of programs. Around 99.0\% of the amino acids of the predicted structure of MAP_PRSO3010 protein were located in the energetically allowed regions for the polypeptide backbone dihedral angle phi $(\psi)$ against psi $(\varphi)$. Thus, the Ramachandran plot revealed that the most dihedral angle of the polypeptide backbone in MAP_PRSO3010 protein structure corresponds to sterically allowed conformations. Similarly, The PROCHECK geometrical descriptors like Chi1-chi2, main chain, side chain, bond length, bond angle and planar groups of the amino acid residues in the predicted protein structure of MAP_PRSO3010 were found to be less deviated from the stable confirmation of the reference structures and, therefore, were found worthy of future structural studies. The supersecondary structural motifs in MAP_PRSO3010 proteins were identified, classified and analyzed using the crystal structure of the Rv0757 protein as a reference. The percentage of supersecondary structural elements in the predicted structure of MAP_PRSO3010 protein were nearly same as that of the template structure (3ROJ). The presence of conserved residues forming the acidic active $\&$ phosphorylation motif as well as an intermolecular recognition \& dimerization motifs positioned at the N-terminal segment of the signal receiver domain depicts that both MAP_PRSO3010 and Rv0757 protein are involved in inter-signal transduction between the receiver and response regulator domains. The presence of conserved winged helix turn helix motif (wHTH) between the two orthologs is another evidence that the wHTH of MAP_PRSO3010 protein is involved in DNA-templated binding and regulation of the various genes belonging to multiple pathways. The Structural elements comprising the wHTH fold of the trans_reg_C domain of both MAP_PRSO3010 and Rv0757 proteins are packed in three-dimensional space via a hydrophobic core, in which the first helix provides a framework for the packing of the remainder domain. The remaining two helices (second and third) and the flexible long loop connecting between them forms the helix-turn-helix motif, with the third helix, forms the recognition helix important for recognition and interaction with the major groove of DNA. The long flexible loop connecting $\alpha 7$ and $\alpha 8$ is slightly disordered and is termed as transactivation loop since the homologous loop in PhoB and OmpR of E.coli interact with the $\alpha$ or $\beta$ subunit of RNA polymerase to initiate transcription while similar interaction for $M t b$ and MAP PhoP is yet to be demonstrated. The $\beta$-hairpin turn at the C-terminal region of the wHTH fold forms the winged motif essential for binding to the minor groove of DNA. A strong positive electrostatic potential was observed at molecular surfaces comprising the wing motif and recognition helix. The occurrence of a positive electrostatic potential in and around the winged helix-turn-helix region of MAP_PRSO3010 protein clearly show the ability of this unique protein fold in nucleotide sequence recognition and DNA binding.

Conclusion. Since in this study a significant homology of sequential, structural and functional is demonstrated between the two orthologs, therefore, it can be postulated that MAP_PRSO3010 a hypothetical protein might play a key role in the coordinated DNA-templated regulation of genes leading to the survival, and virulence of MAP within ruminants and human macrophages. Additional future biochemical and structural studies are required to show the interaction of the MAP_PRSO3010 protein with other virulence factors which, might facilitate MAP in the establishment and propagation of the bacterium within host macrophages. Such studies will increase our current knowledge and understanding of the PPI interactions in MAP and may also contribute to a screening of novel target specific therapeutic against Johne's and Crohn's disease. 


\section{REFERENCE}

[1] Collins, M., Manning, E. (2014). From the history of Johne's disease. Johne's Information Center. http://www.johnes.org/history/index.html.

[2] Dalziel., T.K. (1913). Chronic interstitial enteritis. British Journal of Medicine, 2:2756.

[3] Crohn., B.B, Ginzburg, L., Oppenheimer, G.D. (1932). Regional ileitis-a pathologic and clinical entity. Journal of American Medical Association, 99(16),1323.

[4] Hermon-Taylor, J. (2009). Mycobacterium avium subspecies paratuberculosis, Crohn's disease, and the Doomsday scenario. Gut Pathogen, 1(15): 15.

[5] Sechi, L.A., \& Dow, C.T. (2015). Mycobacterium avium ss. paratuberculosis Zoonosis - The Hundred Year War - Beyond Crohn's Disease. Frontires in Immunology, 6:96.

[6] Li, L., Bannantine, J., Zhang, Q., Amonsin, A., May, B., Alt, D., Banerji, N., Kanjilal, S., Kapur, V. (2005). The complete genome sequence of Mycobacterium avium subspecies paratuberculosis. Proceedings of the National Academy of Sciences of the Unted States, 102(35), 12344-9.

[7] Smith, I. (2003). Mycobacterium tuberculosis Pathogenesis and Molecular Determinants of Virulence. Clinical Microbiology Reviews, 16(3):463-496.

[8] Rappuoli, R., Scarlato, V., Arico, B. (1995). Signal Transduction and Bacterial Virulence. Landes RG Co., Austin, TX

[9] Zahrt, T.C., \& Deretic, V. (2001). Mycobacterium tuberculosis signal transduction system required for persistent infections. Proceedings of the National Academy of Sciences, 98(22), 12706-12711.

[10] Cole, S.T., Brosch, R., Parkhill, J., Garnier, T., Churcher, C., Harris, D., Gordon, S.V., Eiglmeier, K., Gas, S., Barry, C.E., et al. (1998). Deciphering the biology of Mycobacterium tuberculosis from the complete genome sequence. Nature (London), 393, 537-544.

[11] Gonzalo, A.J., Maia, C., Ferrer, N.L., Barilone, N., Laval, F., Soto, C.Y., Winter, N., Daffé, M., Gicquel, B., Martín, C., Jackson, M. (2006). The virulence-associated two-component PhoP-PhoR system controls the biosynthesis of polyketide-derived lipids in Mycobacterium tuberculosis. Journal of Biological Chemistry, 281(3),1313-6.

[12] Walters, S.B, Dubnau, E., Kolesnikova, I., Laval, F., Daffe, M., Smith, I. (2006) The Mycobacterium tuberculosis PhoPR two-component system regulates genes essential for virulence and complex lipid biosynthesis. Molecular Microbiology, 60(2), 312-30.

[13] Asensio, G.J., Soto, C.Y., Arbués, A., Sancho, J., Menéndez, C.M, García, M.J., Gicquel, B., and Martín, C. (2008). The Mycobacterium tuberculosis phoPR Operon is Positively Autoregulated in the Virulent Strain H37Rv. Journal of Bacteriology, 190(21),7068-7078.

[14] Pérez, E., Samper, S., Bordas, Y., Guilhot, C., Gicquel, B., Martín, C. (2001). An essential role for phoP in Mycobacterium tuberculosis virulence. Molecular Microbiology, 41(1), 179-187.

[15] Kuehnel, M.P., Goethe, R., Habermann, A., Mueller, E., Rohde, M., Griffiths, G., Valentin-Weigand, P. (2001). Characterization of the intracellular survival of Mycobacterium avium ssp. paratuberculosis: phagosomal $\mathrm{pH}$ and fusogenicity in $\mathrm{J} 774$ macrophages compared with other mycobacteria. Cell Microbiology, 3, 551-566.

[16] Huerta-Cepas, J., Szklarczyk, D., Forslund, K., Cook, H., Heller, D., Walter, M.C., Rattei, T., Mende, D.R., Sunagawa, S., Kuhn, M., Jensen, L.J., Mering, C.V., Bork, P. (2016). eggNOG 4.5: a hierarchical orthology framework with improved functional annotations for eukaryotic, prokaryotic and viral sequences. Nucleic Acids Research, 44 (D1), D286-D293.

[17] Kyte, J. \& Doolittle, R.F. (1982). A simple method for displaying the hydropathic character of a protein. Journal of Molecular Biology, 157,105-132.

[18] Guruprasad, K., Reddy, B.V.B., Pandit, M.W. (1990). Correlation between the stability of a protein and its dipeptide composition: a novel approach for predicting in vivo stability of a protein from its primary sequence. Protein Engineering, 4,155-161.

[19] Ikai, A.J. (1980). Thermostability and aliphatic index of globular proteins. Journal of Biochemisty, 88,1895-1898.

[20] Gasteiger, E., Hoogland, C., Gattiker, A., Duvaud, S., Wilkins, M.R., Appel, R.D., Bairoch, A. (2005). Protein Identification and Analysis Tools on the ExPASy Server (In): Walker JM (ed) The proteomics Protocols Handbook, Humana Press, pp 571-607

[21] Szklarczyk, D., Franceschini, A., Wyder, S., Forslund, K., Heller, D., Huerta-Cepas, J., Simonovic, M., Roth, A., Santos, A., Tsafou, K.P., Kuhn, M., Bork, P., Jensen, L.J., von Mering, C. (2015). STRING v10: protein-protein interaction networks, integrated over the tree of life. Nucleic Acids Research, 43, D447-52. 
[22] Mitchell, A., Chang, H.Y., Daugherty, L., Fraser, M., Hunter, S., Lopez, R., McAnulla, C., McMenamin, C., Nuka, G., et al. (2015). The InterPro protein families database: the classification resource after 15 years. Nucleic Acids Research, 43 (D1), D213-D221.

[23] Marchler-Bauer, A., Derbyshire, M.K., Gonzales, N.R., Lu, S., Chitsaz, F., Geer, L.Y., Geer, R.C., He, J., Gwadz, M., Hurwitz, .D.I., Lanczycki, C.J., Lu, F., Marchler, G.H.,.Song, J.S., Thanki, N., Wang, Z., Yamashita, R.A., Zhang, D., Zheng, C., Bryant, S.H. (2015). CDD: NCBI's conserved domain database. Nucleic Acids Research, 43, D222-6.

[24] De-Beer, T.A.P., Berka, K., Thornton, J. M., Laskowski, R. A. (2014). PDBsum additions. Nucleic Acids Research, 42, D292-D296.

[25] Nugent, T.\& Jones, D.T. (2013). Membrane protein orientation and refinement using a knowledge-based statistical potential. BMC Bioinformatics, 14, 276.

[26] Van der Lee, R., Buljan, M., Lang, B., Weatheritt, R.J., Daughdrill, G.W., Dunker, A.K., Fuxreiter, M., Gough, J., Gsponer, J., Jones, D.T., Kim, P.M., Kriwacki, R.W., Oldfield, C.J., Pappu, R.V., Tompa, P., Uversky, V.N., Wright, P.E., Babu, M.M. (2014). Classification of Intrinsically Disordered Regions and Proteins. Chemical Reveiws, 114 (13), 6589-66 31.

[27] Biasini, M., Bienert, S., Waterhouse, A., Arnold, K., Studer, G., Schmidt, T., Kiefer, F., Cassarino, T.G., Bertoni, M., Bordoli, L., Schwede, T. (2014). SWISS-MODEL: modeling protein tertiary and quaternary structure using evolutionary information. Nucleic Acids Research, 42 (W1), W252-W258.

[28] Arnold, K., Bordoli, L., Kopp, J., Schwede, T. (2006). The SWISS-MODEL Workspace: A web-based environment for protein structure homology modeling. Bioinformatics, 22,195-201.

[29] Kiefer, F., Arnold, K., Künzli, M., Bordoli, L., Schwede, T. (2009). The SWISS-MODEL Repository and associated resources. Nucleic Acids Research, 37, D387-D392.

[30] Guex, N., Peitsch, M.C., Schwede, T. (2009).Automated comparative protein structure modeling with SWISS-MODEL and Swiss-PdbViewer: A historical perspective. Electrophoresis, 30(S1), S162-S173.

[31] Sali, A., \& Blundell, T.L. (1993). Comparative protein modeling by satisfaction of spatial restraints. J Molecular Biology, 234, 779-815.

[32] Guex, N., Peitsch, M.C. (1997). SWISS-MODEL and the Swiss-PdbViewer: an environment for comparative protein modeling. Electrophoresis,18, 2714-2723.

[33] Benkert, P., Kunzli, M., Schwede, T. (2009). QMEAN server for protein model quality estimation. Nucleic Acids Research, 37, W510-514.,

[34] Benkert, P., Biasini, M., Schwede, T. (2011). Toward the estimation of the absolute quality of individual protein structure models. Bioinformatics, 27, 343-350.

[35] Melo, F., \& Feytmans, E. (1998). Assessing protein structures with a non-local atomic interaction energy. Journal of Molecular Biology, 277(5),1141-1152.

[36] Van Gunsteren, W.F, Billeter, S.R. et al. (1996). Biomolecular Simulations: The GROMOS96 Manual and User Guide. Zürich, VdF Hochschulverlag ETHZ.

[37] Zhou, H., Zhou, Y. (2002). Distance-scaled, finite ideal gas reference state improves structure-derived potentials of mean force for structure selection and stability prediction. Protein Science, 11, 2714-2726.

[38] Laskowski, R.A., MacArthur, M.W., Moss, D.S., Thornton, J.M. (1993). PROCHECK: A program to check the stereochemical quality of protein structures. Journal of Applied Crystalography, 26, 283-291.

[39] Via, L.E., Curcic, R., Mudd, M.H., Dhandayuthapani, S., Ulmer, R.J., Deretic, V. (1996). Elements of signal transduction in Mycobacterium tuberculosis: in vitro phosphorylation and in vivo expression of the response regulator MtrA. Journal of Bacteriology, 178, 3314-3321.

[40] Yarwood, J.M., McCormick, J.K., Schlievert, P.M. (2001). Identification of a novel two-component regulatory system that acts in global regulation of virulence factors of Staphylococcus aureus. Journal of Bacteriology, 183,1113-1123.

[41] Wren, B.W., Colby, S.M., Cubberley, R.R., Pallen, M.J. (1992). Degenerate PCR primers for the amplification of fragments from genes encoding response regulators from a range of pathogenic bacteria. FEMS Microbiology Letters, 78(2-3), 287-291.

[42] Bretl, D.J., Demetriadou, C., Zahrt, T.C. (2011). Adaptation to environmental stimuli within the host: two-component signal transduction systems of Mycobacterium tuberculosis. Microbiology Molecular Biology Review. 75(4), 566-582.

[43] Parish, T., Smith, D.A., Roberts, G., Betts, J., Stoker, N.G. (2003). The senX3-regX3 two-component regulatory system of Mycobacterium tuberculosis is required for virulence. Microbiology, 149, 14231435. 
[44] Haydel, S.E., Clark-Curtiss, J.E. (2004). Global expression analysis of two-component system regulator genes during Mycobacterium tuberculosis growth in human macrophages. FEMS Microbiology Letters, 236, 341-347.

[45] Engohang-Ndong, J., Smith, I.(2007). Structure of the DNA-binding domain of the response regulator PhoP from Mycobacterium tuberculosis. Biochemistry, 46(51), 14751-14761.

[46] Nowak, E., Panjikar, S., Konarev, P., Svergun, D.I., Tucker, P.A. (2006). The structural basis of signal transduction for the response regulator PrrA from Mycobacterium tuberculosis. Journal of Biological Chemistry, 281, 9659-9666.

[47] Babu, M.M., Lee, V.D.R., Groot, N.S.D., Gsponer, J. (2011). Intrinsically disordered proteins: regulation and disease. Current Opinion in Structural Biology, 21,1-9. 